\title{
Further positive transfer effects obtained through injections of brain homogenates'
}

\author{
JAMES A. DYAL ${ }^{2}$ AND ARNOLD M. GOLUB ${ }^{3}, T E X A S$ \\ CHRISTIAN UNIVERSITY, Fort Worth, Texas
}

Two experiments in a series of four experiments testing for the "transfer via injection" effect are reported. Only one of the experiments yielded positive results: however, the combined probability for the series of experiments using the same procedure is highly significant. It is suggested that both activation and specificity are involved in positive transfer effects.

Considerable controversy has arisen concerning the validity of the phenomenon of transfer of response bias from a trained organism to an untrained organism through the injection of brain homogenates or extracts. Experiments 1 and 2 in the present series reported significant behavioral transfer effect (Dyal, Golub, \& Marrone, 1967). However, since experimental reproducibility has become a critical issue in this research area, two further experiments were conducted using essentially the same procedures as Experiments 1 and 2.

\section{EXPERIMENT 3}

Method. The apparatus was the same operant conditioning equipment used in Experiments 1 and 2. The Ss were 56 male Sprague-Dawley rats between 100 and 150 days old. Seven Ss were dropped from the experiment for failure to learn or procedural error. The Ss were randomly assigned to two donor groups (AER and AER-Y, N $=16$ ) and three recipient groups. The experimental donors (Group AER) were given eight daily training sessions under a CRF schedule, followed by three extinction sessions, followed in turn by four sessions of reacquisition of the bar press response on a CRF schedule. Two events were contingent on a bar press during CRF, the delivery of a 45 $\mathrm{mg}$ Noyes pellet and the simultaneous diminution of the photoelectric beam across the food cup and a click of the food delivery mechanism. During extinction a bar press resulted in the lightclick stimulation only.

The control donors (Group AER-Y) never experienced a contingency between bar pressing and the events involved in food delivery. They were, however, yoked to the experimental donors in such a way that they received the light-click event in their chamber whenever the experimental $S$ pressed the bar. When a control $\mathrm{S}$ pressed the bar the event was recorded on a counter but the pellet dispenser was not pulsed, and thus no pellet or light-click stimulation was received. All donors were $23 \mathrm{~h}$ deprived of food at the time of their 30 min training session.

Within $1 \mathrm{~h}$ after the completion of training on the last day of training, the donors were killed with ether. The brain, excluding the olfactory bulbs and the cerebellum, was removed within $2 \mathrm{~min}$ of death. The homogenization and injection procedure was the same as that previously reported in Experiments 1 and 2.

The recipients were placed on a $23 \mathrm{~h}$ food deprivation schedule for six days prior to testing for operant levels of magazine entry ( $\mathrm{ME}=$ the number of occasions on which $\mathrm{S}$ stuck its nose into the food magazine). During this test Ss were placed into the experimental chamber and presented with the light-click stimulus every $60 \mathrm{sec}$ for $30 \mathrm{~min}$. The number of $\mathrm{ME}$ were accumulated on counters. The Ss were $48 \mathrm{~h}$ deprived during this pre-injection operant level test. They were maintained on the $23 \mathrm{~h}$ food deprivation schedule for 16 more days (while the donors were being trained). Each recipient was then injected intraperitoneally with $3.2 \mathrm{ml}$ of either brain homogenate from AER donors, brain homogenate from AER-Y donors, or physiological saline. They were tested $24 \mathrm{~h}$ later. The first 30 min test was identical to the operant baseline tests described above. During the second 30 min test the bar was activated on a CRF schedule, and both ME and bar presses (BP) were recorded.

Results. The experimental recipients did not differ from control recipients on either BP or ME measures during either the first or the second $30 \mathrm{~min}$ test sessions. When pre- vs post-injection scores were compared the saline group showed a significant increase $(p=.031)$ in ME during the first $30 \mathrm{~min}$ session and the control group a significant increase during the second test session $(\mathrm{p}=.016)$.

The results of Experiment 3 are uniformly negative with regard to a demonstration of the transfer via injection phenomenon. After surveying the present experimental procedure for changes in parameters which might explain the difference between Experiment 3 and Experiments 1 and 2, it was felt that the prime factor might be that Experiment 3 was conducted by relatively untrained undergraduate assistants whereas Experiments 1 and 2 had been conducted by more sophisticated graduate students. It was thus decided to repeat Experiment 3 with the same undergraduate Es, who were now presumably better trained having been through the entire procedure in Experiment 3.

\section{EXPERIMENT 4}

Method and Results. The procedure in Experiment 4 was identical to that of Experiment 3 except that the recipients were placed on a $23 \mathrm{~h}$ food deprivation schedule for five days prior to collection of pre-injection operant levels, and both $\mathrm{BP}$ and ME data were collected. Following the pre-injection tests $\mathrm{Ss}$ were returned to an ad lib feeding schedule until five days prior to the post-injection test. The interval between pre- and post-injection tests ranged from $9-16$ days.

No saline control group was run in Experiment 4. Thirty donor Ss were assigned at random to Training Groups AER and AER-Y; the recipient groups contained six and seven Ss, respectively. The mean post-injection ME was 196 and 138 for Recipient Groups AER and AER-Y; the pre-injection means were 66 and 75. Both Groups AER and AER-Y manifested significant increases over pre-injection levels; however, Group AER increased significantly more than Group AER-Y $(U=8, p=.037)$.

There were no significant differences between the AER and AER-Y groups in the pre- vs post-injection BP difference scores. The pre-injection BP mean was 11.2 for the two groups combined; the comparable post-injection score was $5.3(\mathrm{Mdn}=8.0$ and 1.0 , respectively). Ten of the 12 recipients made fewer $\mathrm{BP}$ on the post-injection test $(\mathrm{X}=2, \mathrm{p}<.033$, sign test $)$.

There were no reliable differences between AER and AER-Y groups on either $\mathrm{BP}$ or $\mathrm{ME}$ during the second test period.

\section{DISCUSSION}

The results of Experiment 4 are consistent with Experiments 1 and 2 in demonstrating a significant transfer via injection effect when ME during the first 30 min test session is the dependent variable. Since all four experiments in this series used essentially the same procedures to test the same hypothesis, a test was run to determine the combined probability of the effect (Winer, 1962). The resulting chi square statistic (25.43) was significant beyond the .001 level. We may thus conclude that, using these procedures, the transfer via injection effect is both statistically reliable and experimentally reproducible.

Three possible interpretations of the transfer via injection phenomenon may be proposed. First, it could be suggested that transfer does not involve "learning" at all but is in fact a stimulus sensitization effect. We believe that this interpretation is not applicable to our data since the yoked control donors received the same sensory stimulation as did the experimental donors. Second, the effect could be due to a differential transfer of general activation. Although the present experiment cannot clearly refute such an interpretation, the post-injection $\mathrm{BP}$ rate, which was significantly lower than the pre-injection rate, strongly argues against its validity.

The third interpretation is that some specific learning or memory factor is being transferred. Although this series of experiments cannot definitely support or refute this interpretation, the results of Experiments 3 and 4 suggest that both a general activation and some specific memory factor may contribute to positive results. It will be recalled that in Experiment 3 there was no increase in ME performance over baseline for either Group AER or AER-Y, and that, furthermore, no significant transfer effect was obtained. On the other hand, in Experiment 4 where a differential transfer effect was obtained both groups showed a significant elevation of performance over pre-injection baselines. However, in order to clearly implicate a specific memory factor some variety of discrimination task is required. The results 
of two discrimination experiments in our laboratory tend to support the contention that both activation and specific transfer of information are involved in positive transfer effects (Dyal \& Golub, 1967; Dyal \& Golub, in press).

\section{REFERENCES}

DYAL, J. A., \& GOLUB, A. M. An attempt to obtain shifts in brightness preference as a function of injection of brain homogenate. J. biol. Psychol., 1967, 9, 29-33.

DYAL, J. A., \& GOLUB, A. M. Behavioral transfer via injection of brain homogenate: Activation or specificity? In W. Byrne (Ed.), Molecular approaches to learning and memory. AAAS symposium. In press.

DYAL, J. A., GOLUB, A. M., \& MARRONE, R. L. Transfer effects of intraperitoneal injection of brain homogenates. Nature, 1967, 214, 720-721.

WINER, B. J. Statistical principles in experimental design. New York: McGraw-Hill, 1962.

\section{NOTES}

1. This research was supported by a grant from the Texas Christian University Research Foundation. The authors thank Ernest Harrell, K'lee Hetherington, David Ogrod, John Schmadl, and Harold Winer who collected data for Experiments 3 and 4.

2. Currently National Science Foundation Science Faculty Fellow at the University of California, Berkeley.

3. Currently post-doctoral Fellow at the Mental Health Research Institute, University of Michigan. 